\title{
Epidermal Growth Factor-Like Immunoreactive Substance(s) in Human Platelets
}

\author{
Noriaki Tokida, ${ }^{1}$ Masayuki Kurobe, ${ }^{2}$ Shoei Furukawa, ${ }^{3}$ \\ and Kyozo HaYASHI ${ }^{1, *}$ \\ ${ }^{1}$ Department of Pharmaceutics, Gifu Pharmaceutical University, \\ Mitahora-Higashi, Gifu 502, Japan \\ ${ }^{2}$ Gifu College of Medical Technology, Ichihiraga, Seki 501-32, Japan \\ ${ }^{3}$ Division of Immunology, National Center of Neurology and \\ Psychiatry, Kodaira, Tokyo 187, Japan
}

(Received October 1, 1987)

\begin{abstract}
Summary Some properties of epidermal growth factor (EGF)-like immunoreactive substances (EGF-LI) found in human platelets were studied. Human platelets were treated with acidic, alkaline, or hypotonic solutions or by freezing-thawing. Then after centrifugation the hEGF-LI in the resulting supernatant was examined by our rapid enzyme immunoassay system for the presence of hEGF-LI's released from the platelets. The amount of hEGF-LI released from platelets varied with the treatment, the alkaline or freezing-thawing treatment being the most effective in liberating the hEGF-LI. The hEGF-LI was applied to a column of Sephadex G-100 and was detected in four fractions showing molecular weights of about 280,56, 20, and 6 kdaltons. The high molecular weight hEGF-LI's having molecular weights of 280 and $56 \mathrm{kD}$ were converted to $20 \mathrm{kD}$ one by treatment with 2-mercaptoethanol. So it appears that some of the hEGF-LI's in platelets may exist in a complex form through disulfide linkages with some proteins in the platelets.
\end{abstract}

Key Words: epidermal growth factor, human platelets, liberation, biochemical properties, complex form

Epidermal growth factor (EGF), a polypeptide having a molecular weight of 6,045 , stimulates proliferation and differentiation of a wide variety of cells [1-3]. While EGF is thought to play a role in almost every tissue in the body both during development and in the adult, the exact nature of this role is not clear.

The inability to experimentally lower serum levels of EGF in mice by removing the submaxillary glands [4], the major site of EGF synthesis in the mouse [5], has

*To whom correspondence should be addressed. 
hampered clarification of the role of EGF. This failure to alter EGF levels surgically is probably due to EGF's being synthesized at several anatomical sites in the body [6-8], where the factor functions more in a paracrine rather than in an endocrine manner.

The fact that growth of various cells in vitro requires the addition of serum to the medium suggests that there exist potent mitogenic factors in serum or factors which are liberated during the process of blood coagulation. The presence of EGF or EGF-like immunoreactive material (hEGF-LI) in platelets has already been reported [7], and the data suggest that EGF or EGF-LI from platelets may function not only in regulating cell proliferation and/or differentiation in vivo but also in wound healing. The present report describes some properties of hEGF-LI in human platelets.

\section{MATERIALS AND METHODS}

Materials. Human epidermal growth factor (hEGF) purified from human urine by the method of Nishimuro et al. [9] was generously provided by Japan Chemical Research Co., Ltd. (Kobe) and was used as the standard in the enzyme immunoassay (EIA) system for hEGF.

Two-site enzyme immunoassay. EIA system for hEGF and hEGF-LI was carried out by using anti-hEGF antibody IgG-coated polystyrene beads and antihEGF Fab'-linked peroxidase (Sigma, horseradish, EC 1.11.1.7, St. Louis, MO) as described previously [10].

Samples. Human sera and plasma obtained from healthy volunteers were kindly provided by Gifu Red Cross Blood Bank (Gifu).

(1) Preparation of washed platelets: Out-dated platelet-rich plasma (one unit, one to four days out-dated) was centrifuged at $500 \mathrm{rpm}$ for $5 \mathrm{~min}$ at $5^{\circ} \mathrm{C}$. The resulting pellets were washed three times with $10 \mathrm{ml}$ of CPD solution (Japan Pharmacopeia XI, containing $89.4 \mathrm{~mm}$ sodium citrate, $15.6 \mathrm{~mm}$ citric acid, 128.8 $\mathrm{mm}$ glucose, and $16.1 \mathrm{~mm}$ sodium dihydrogen phosphate). The washed pellets were suspended in $5 \mathrm{ml}$ of CPD solution and subjected to the treatments described below as the washed platelets.

(2) Treatment of platelets with acidic, alkaline, or hypotonic solutions: Aliquots $(0.8 \mathrm{ml}$ each) of platelet suspension, freshly prepared, were centrifuged at 3,500 $\mathrm{rpm}$ for $20 \mathrm{~min}$ at $5^{\circ} \mathrm{C}$. The resulting pellets were resuspended in a $0.5 \mathrm{ml}$ volume of either saline, distilled water, $1 \%$ acetic acid, $0.1 \mathrm{M}$ Tris(hydroxymethyl)aminomethane, or $0.1 \mathrm{M}$ sodium carbonate solution and left to stand overnight at $5^{\circ} \mathrm{C}$. Then, these suspensions were centrifuged at $100,000 \times g$ for $30 \mathrm{~min}$ at $5^{\circ} \mathrm{C}$. The resulting supernatants were used as the platelet extracts.

(3) Platelet treatment by freezing-thawing: The washed platelets were suspended in $0.5 \mathrm{ml}$ of CPD solution and treated with five cycles of freezing-thawing to fracture the platelets completely. The resulting suspension was then treated in the same manner as described in (2). 
Size-exclusion chromatography. All chromatographic operations were carried out at ambient temperature, at a constant flow rate of $1.0 \mathrm{ml}$ per min, and with $50 \mathrm{~mm}$ phosphate buffer, $\mathrm{pH}$ 7.0, containing $100 \mathrm{~mm}$ sodium chloride as the eluent. The chromatographic system employed consisted of a Jasco Tri-Rotor Pump (Japan Spectroscopic Co., Ltd., Tokyo) and TSK-G 3000 SW column (i.d. $7.5 \times 600 \mathrm{~mm}$, Toyo Soda Co., Ltd., Tokyo).

\section{RESULTS}

\section{hEGF-LI levels in human plasma and serum}

hEGF levels in plasma and serum of 40 healthy volunteers were determined by our rapid EIA system for hEGF using 10- $\mu$ l samples. As shown in Fig. 1, the mean levels of hEGF-LI in plasma and sera were 0.29 and $1.10 \mathrm{ng} / \mathrm{ml}$, respectively. Namely, the hEGF level in sera was about four-fold higher than that of plasma, suggesting that hEGF-LI was released from the platelets during blood coagulation. So, we examined various means of liberating hEGF-LI from human platelets.

\section{Liberation of hEGF-LI from human platelets by various treatments}

The amounts of hEGF-LI released from human platelets by various treatment were measured by our EIA system for hEGF. The mean values of hEGF-LI levels in the various extracts ( 6 samples for each treatment) are shown in Table 1.

Hypotonic treatment yielded over twice the amount of hEGF-LI found in the saline extract. However, when human platelets were treated by freezing-thawing or with alkaline solution, such as $0.1 \mathrm{~m}$ Tris(hydroxymethyl)aminomethane or sodium carbonate solution, an even higher level of hEGF-LI was found in the extract. On the contrary, only a small amount of hEGF-LI was liberated by the acidic treatment. The $\mathrm{pH}$ of $0.1 \mathrm{~m}$ Tris(hydroxymethyl)aminomethane or sodium carbonate solution is about 10, and so the freezing-thawing treatment was used in the following experiment considering the poor stability of hEGF-LI under such basic conditions.

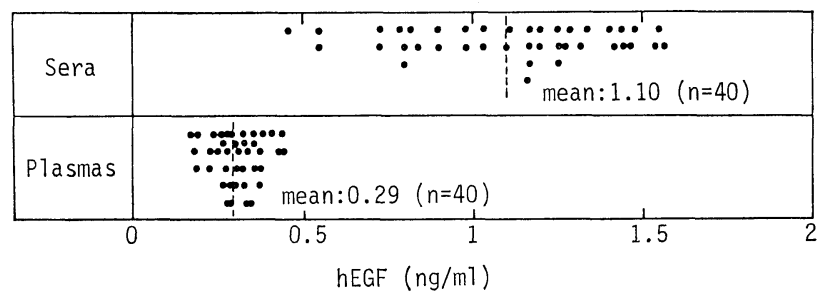

Fig. 1. Levels of hEGF-LI in normal human serum and plasma. Forty samples from healthy volunteers were used for each experiment. Each point indicates the mean of duplicate assays, and the dotted lines indicate the average level of hEGF of the samples. 
Table 1. Amounts of hEGF-LI in extracts of human platelets prepared by various treatments.

\begin{tabular}{|c|c|c|c|c|c|c|c|}
\hline \multirow{3}{*}{ Treatment } & \multicolumn{6}{|c|}{ hEGF-LI (pg/ml) } & \multirow{3}{*}{ mean $\pm S D$} \\
\hline & \multicolumn{6}{|c|}{ Sample No. } & \\
\hline & 1 & 2 & 3 & 4 & 5 & 6 & \\
\hline Freezing-thawing & 405 & 539 & 455 & 540 & 396 & 281 & $436 \pm 98$ \\
\hline Saline & 131 & 86 & 79 & 113 & 92 & 46 & $92 \pm 29$ \\
\hline $\mathrm{H}_{2} \mathrm{O}$ & 288 & 280 & 286 & 216 & 187 & 166 & $237 \pm 54$ \\
\hline $1 \%$ Acetic acid & 17 & 13 & 15 & 11 & 10 & 12 & $13 \pm 3$ \\
\hline $0.1 \mathrm{M}$ Tris & 643 & 692 & 540 & 505 & 471 & 452 & $551 \pm 97$ \\
\hline $0.1 \mathrm{M} \mathrm{Na} \mathrm{Na}_{3}$ & 585 & 693 & 500 & 553 & 598 & 455 & $564 \pm 83$ \\
\hline
\end{tabular}

Details of the procedures used are described in Materials and Methods. A 100- $\mu$ l aliquot of each extract was subjected to the EIA for hEGF.

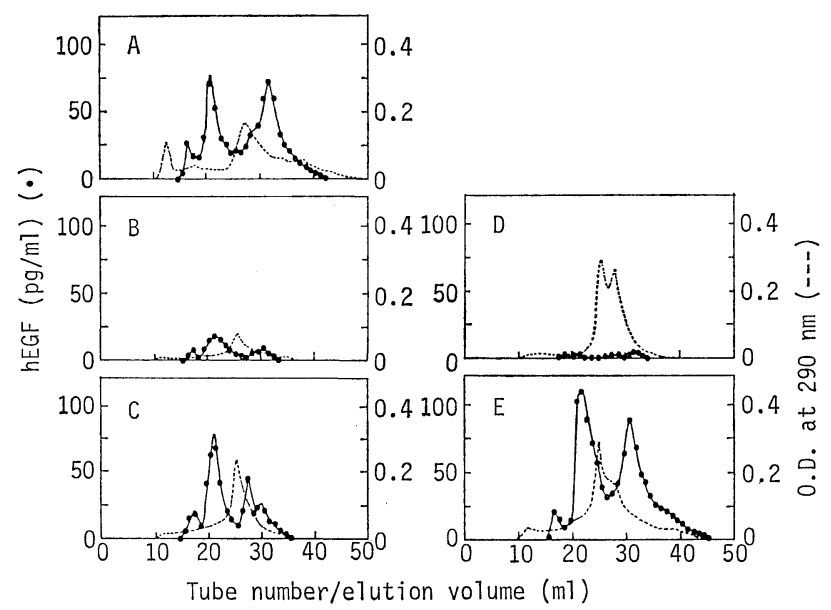

Fig. 2. Size-exclusion chromatograms of the platelet extracts obtained by various treatments. A $200-\mu 1$ sample of each extract was applied to a size-exclusion chromatographic column (TSK-G $3000 \mathrm{SW}$ column, $7.5 \times 600 \mathrm{~mm}$ ) equilibrated with $50 \mathrm{~mm}$ phosphate buffer, $\mathrm{pH} 7.0$, containing $100 \mathrm{~mm} \mathrm{NaCl}$, and then eluted with the same buffer at a rate of $1.0 \mathrm{ml}$ per min. Fractions of $1.0 \mathrm{ml}$ each were collected. A $100-\mu 1$ aliquot of each fraction was subjected to the EIA for hEGF. A, Freezing and thawing; B, saline; $\mathrm{C}, \mathrm{H}_{2} \mathrm{O}$; $\mathrm{D}, 1 \%$ acetic acid; $\mathrm{E}, 0.1 \mathrm{M} \mathrm{Na} \mathrm{NO}_{3}$.

Size-exclusion chromatography of the platelet extracts prepared by various treatments

Each extract prepared by one of the various methods was applied to a sizeexclusion chromatographic column. As shown in Fig. 2, four peaks with immunodetectable hEGF-LI appeared at approximately tube Nos. 18, 22, 28, and 32 when the sample had been subjected to freezing-thawing. But the peak sizes and 
number of peaks were different for the other extracts. The sizes of peaks emerging near tube Nos. 22 and 32 were almost the same by gel filtration of the sample obtained by freezing-thawing, but the size of the peak emerging near tube No. 22 was larger than that near 32 in the extract of the platelets treated with hypotonic or alkaline solution. The hypotonic treatment extract contained much more hEGFLI emerging near tube No. 28 than the analogous peak found in the extracts prepared by the other treatments.

A large amount of protein was liberated from the platelets by acidic treatment, but only a small amount of hEGF-LI was detected. In view of these results, it is likely that hEGF-LI in platelets exists as a complex with some other proteins.

\section{Some properties of the hEGF-LI in human platelets}

The molecular weight of each of the four hEGF-LI's liberated from the human platelets was estimated by gel filtration on a Sephadex G-100 column. As shown in Fig. 3, peaks of hEGF-LI were observed near tube Nos. 25, 38, 49, and 57. By comparing the elution positions of the latter three with those of standard proteins, the molecular weights of these hEGF-LI's were estimated to be approximately 56, 20 and $6 \mathrm{kD}$, respectively. But it was difficult to precisely estimate the molecular weight of the first peak by this column as it appeared near the void volume; therefore its molecular weight was estimated by gel filtration using a Sephadex G-150 column and appeared to be about $280 \mathrm{kD}$.

Treatment of hEGF-LI's in human platelets with 2-mercaptoethanol

We have already reported that the high molecular weight hEGF-LI (HMW-

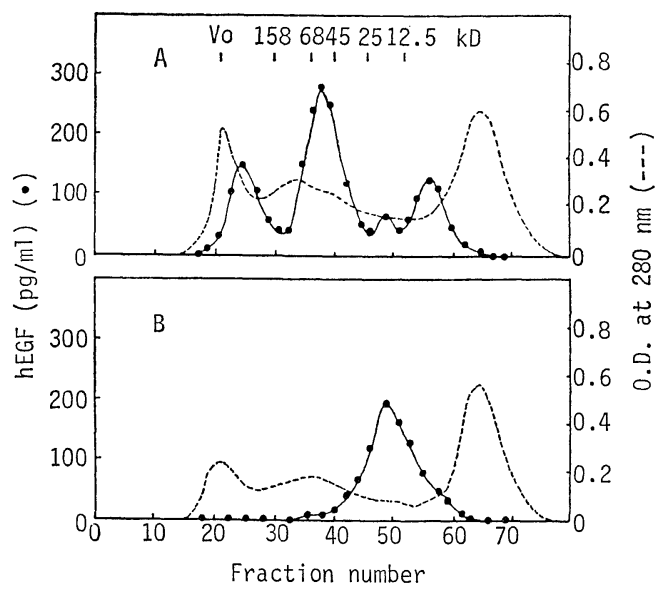

Fig. 3. Sephadex G-100 elution profiles of hEGF-LI's in human platelet extract prepared by freezing-thawing. One milliliter of human platelet extract (A) or $50 \mathrm{~mm}$ 2-mercaptoethanol-treated extract (B) was applied to a Sephadex G-100 column $(1.2 \times 66.0 \mathrm{~cm})$ equilibrated with $50 \mathrm{~mm}$ phosphate buffer, $\mathrm{pH} 7.42$, containing $77 \mathrm{~mm} \mathrm{NaCl}$, and then eluted with the same buffer at a rate of $4 \mathrm{ml}$ per hour. Fractions of $1.0 \mathrm{ml}$ each were collected, and $100 \mu 1$ of each fraction was subjected to EIA. 
hEGF-LI) in plasma is converted by reduction with 2-mercaptoethanol to a form eluting identically as standard hEGF [11], which results suggest that a considerable amount of hEGF-LI in human platelets may exist as a HMW-form through S-S linkage with some macromolecule(s). Thus, we examined whether or not hEGF-LI in platelets exists in a covalently associated form with other protein(s) through disulfide bridge(s). So, an extract obtained by freezing-thawing of platelets was treated with $50 \mathrm{~mm}$ 2-mercaptoethanol overnight at $2^{\circ} \mathrm{C}$ prior to its application to a Sephadex G-100 column and was then gel-filtered on the column with $50 \mathrm{~mm}$ phosphate buffer, $\mathrm{pH} 7.42$, containing $77 \mathrm{~mm} \mathrm{NaCl}$ as eluent. As shown in Fig. 3, one symmetrical peak with a minor shoulder near the position at which hEGF elutes was observed at the elution position indicating a molecule of molecular weight $20 \mathrm{kD}$. From these results, the HMW-hEGF-LI's in human platelets appear to exist as a hEGF-LI protein complex formed through disulfide linkages, as is the case for hEGF-LI in plasma. However, the molecular weight of hEGF-LI in platelets seems to be higher than that of plasma hEGF in having a molecular weight of about $20 \mathrm{kD}$ compared with the $6 \mathrm{kD}$ of plasma or standard hEGF.

\section{DISCUSSION}

In the blood coagulation process, platelets make a thrombus at the site of injury on the vascular wall, and, besides, they release various kinds of growth factors. As already reported, human epidermal growth factor-like immunoreactive substances (hEGF-LI) are present in human platelets [12]. However, detailed studies concerning their nature have not been carried out.

In this study, we found that four hEGF-LI's having molecular weights of about $280,56,20$, and $6 \mathrm{kD}$ are liberated from human platelets by various forms of treatment, such as freezing-thawing, alkali, acid, etc. Kurobe et al [11] have reported that the high molecular weight hEGF-LI (HMW-hEGF-LI's) in human plasma can be converted to standard hEGF having a molecular weight of about $6 \mathrm{kD}$ by treatment with 2-mercaptoethanol. In the present study HMW-hEGFLI's from human platelets having molecular weights of about 280 and $56 \mathrm{kD}$ were also converted to a lower molecular weight form by the same treatment; however, this molecular weight was considerably higher than that of standard hEGF, being $20 \mathrm{kD}$. The reason for this difference is not known; but it is clear that considerable amounts of hEGF-LI in human platelets exist as a HMW-form held together through S-S linkages with some macromolecule(s) in the platelets.

The physiological significance of hEGF-LI's in human platelets is still unknown, though they are considered to be released during the blood coagulation process and to play important roles in wound healing. Detailed biochemical properties of the platelet hEGF-LI's that form a complex with other platelet component(s) still remain to be clarified. 
This work was aided in part by Grants-in-Aid for Cancer Research from the Ministry of Health and Welfare of Japan, and by an Itoh Foundation Research Grant for 1987. Authors also thank to Miss K. Tanno of the Gifu Red Cross Blood Bank for providing the blood samples.

\section{REFERENCES}

1. Carpenter, G., and Cohen, S. (1979): Epidermal growth factor. Annu. Rev. Biochem., 48, 193-216.

2. Cohen, S., and Taylor, J.M. (1974): Epidermal growth factor; chemical and biological characterization. Recent Prog. Horm. Res., 30, 511-532.

3. Cohen, S., and Savage, C.R., Jr. (1974): Recent studies on the chemistry and biology of epidermal growth factor. Recent Prog. Horm. Res., 30, 533-550.

4. Byyny, R.L., Orth, D.N., Cohen, S., and Doyne, E.S. (1974): Epidermal growth factor; effects of androgens and adrenergic agents. Endocrinology, 95, 776-782.

5. Cohen, S. (1962): Isolation of mouse submaxillary gland protein accelerating incisor eruption and eyelid opening in the newborn animal. J. Biol. Chem., 237, 1555-1562.

6. Hirata, Y., and Orth, D.N. (1979): Epidermal growth factor (urogastrone) in human tissues. J. Clin. Endocrinol. Metab., 46, 929-936.

7. Oka, Y., and Orth, D.N. (1983): Human epidermal growth factor/ $\beta$-urogastrone is associated with blood platelets. J. Clin. Invest., 72, 249-259.

8. Kasselberg, A.G., Orth, D.N., Gray, M.E., and Stahlman, M.T. (1985): Immunocytochemical localization of human epidermal growth factor/urogastrone in several human tissues. $J$. Histochem. Cytochem., 33, 315-322.

9. Nishimuro, S., Nishimura, T., Uchida, N., Shirono, H., and Hiratani, H. (1985): Heterogeneity of human epidermal growth factor/urogastrone from human urine. Chem. Pharm. Bull., 33, 4037-4040.

10. Kurobe, M., Tokida, N., Furukawa, S., Ishikawa, E., and Hayashi, K. (1986): Development of a sensitive enzyme immunoassay for human epidermal growth factor (Urogastrone). Clin. Chim. Acta, 156, 51-60.

11. Kurobe, M., Furukawa, S., and Hayashi, K. (1986): Molecular nature of human epidermal growth factor (hEGF)-like immunoreactivity in human plasma. Biochem. Int., 12, 677-683.

12. Kurobe, M., Tokida, N., Furukawa, S., and Hayashi, K. (1986): Some properties of human epidermal growth factor (EGF)-like immunoreactive material originating from platelets during blood coagulation. Biochem. Int., 13, 729-733. 